\title{
Aplicação do Bosque Modelo para soluções de conflitos nas ações de gestão participativa do território da Borborema-Paraíba, Brasil
}

\author{
Application of the Model Forest for conflict resolution in participatory management actions in the \\ territory of Borborema-Paraíba, Brazil \\ Aplicación del Bosque Modelo para la resolución de conflictos en acciones de manejo participativo \\ en el territorio de Borborema-Paraíba, Brasil
}

\author{
Amanda Cristiane Gonçalves Fernandes \\ ORCID: https://orcid.org/0000-0001-8462-6171 \\ Universidade Federal de Campina Grande, Brasil \\ E-mail: amandafernandestt@gmail.com \\ Igo Marinho Serafim Borges \\ ORCID: https://orcid.org/0000-0002-3662-1859 \\ Universidade Federal de Campina Grande, Brasil \\ E-mail: igomarinho27@gmail.com \\ Viviane Farias Silva \\ ORCID: https://orcid.org/0000-0002-5891-0328 \\ Universidade Federal de Campina Grande, Brasil \\ E-mail: viviane.farias@professor.ufcg.edu.br \\ Jean Oliveira Campos \\ ORCID: https://orcid.org/0000-0002-2874-754X \\ Universidade Federal da Paraíba, Brasil \\ E-mail: jeannolliveira@gmail.com \\ Anderson Felipe Leite dos Santos \\ ORCID: https://orcid.org/0000-0002-1947-5175 \\ Universidade Estadual Paulista Júlio de Mesquita Filho, Brasil
E-mail: anderson.felipe@unesp.br \\ Emanuelly Cristovão Barbosa da Silva \\ ORCID: https://orcid.org/0000-0003-3835-1132 \\ Universidade Estadual da Paraíba, Brasil \\ E-mail: cristovamemanuelly@gmail.com \\ Cleibson Lima Silva \\ ORCID: https://orcid.org/0000-0002-0663-8828 \\ Centro Universitário Maurício de Nassau, Brasil \\ E-mail: cleibson.cls@gmail.com \\ Joaquim Rangel Andrade da Silva \\ ORCID: https://orcid.org/0000-0002-8183-1126 \\ Universidade Estadual da Paraíba, Brasil \\ E-mail: rangelandrade83@gmail.com \\ Iury Gomes Silva \\ ORCID: https://orcid.org/0000-0002-9537-7091 \\ Universidade Estadual da Paraíba, Brasil \\ E-mail:iurygomes86@gmmail.com \\ Miriam Souza Martins \\ ORCID: https://orcid.org/0000-0002-3512-4770 \\ Universidade Federal de Campina Grande, Brasil \\ E-mail:miriam2009souza@gmail.com \\ Magna Jussara Rodrigues Santos \\ ORCID: https://orcid.org/0000-0001- 8026-6607 \\ Universidade Estadual da Paraíba, Brasil \\ E-mail: magna.santos@aluno.uepb.edu.br
}

\begin{abstract}
Resumo
Uma das características principais do semiárido brasileiro é a variabilidade climática e a escassez de água. Conviver com o semiárido mesmo com todo o seu potencial naturalístico e cultural é um desafio, devido os prolongados períodos de estiagens e a limitação dos recursos hídricos. O presente estudo visa adequar o Bosque Modelo como metodologia de solução de conflitos nas ações de gestão participativa no território do Pólo da Borborema para o fortalecimento das coalisões e medidas estratégicas de convivência para com o semiárido. Para tanto, usou-se a metodológica de Bosque
\end{abstract}


como modelo utilizada para solucionar conflitos através de um recorte espacial ou paisagístico de um cenário em desequilíbrio ambiental e social. Sendo assim, os principais resultados obtidos através da aplicação do modelo bosque foram as diversas alternativas possíveis para solução de conflitos através da mobilização social com diversos interesses e consequentemente o fortalecimento de medidas que visem atenuar os efeitos do processo de desertificação em uma região com escassez de recursos naturais e que vive um processo de extinção de seu bioma, a Caatinga. Conclui-se portanto que, foi possível obter uma melhor visualização da gestão participativa no território da Borborema. É preciso destacar que os possíveis conflitos que surgem dos diferentes grupos passam a ser discutidos também como medidas de eficácia da gestão participativa.

Palavras-chave: Semiárido; Mobilização social; Coalisões.

\begin{abstract}
One of the main characteristics of the Brazilian semiarid region is climate variability and water scarcity. Living with the semiarid region, even with all its naturalistic and cultural potential, is a challenge, due to the long periods of drought and the limitation of water resources. This study aims to adapt the Model Forest as a conflict solution methodology in participatory management actions in the territory of the Borborema Pole to strengthen coalitions and strategic measures of coexistence with the semiarid region. Therefore, the Bosque methodology was used as a model used to solve conflicts through a spatial or landscape cut of a scenario in environmental and social imbalance. Thus, the main results obtained through the application of the forest model were the various possible alternatives for conflict resolution through social mobilization with different interests and, consequently, the strengthening of measures aimed at mitigating the effects of the desertification process in a region with a shortage of natural resources and is experiencing a process of extinction in its biome, the Caatinga. It is therefore concluded that it was possible to obtain a better view of participatory management in the territory of Borborema. It should be noted that the possible conflicts that arise from different groups are also discussed as measures of the effectiveness of participatory management.
\end{abstract}

Keywords: Semiarid; Social mobilization; Coalitions.

\title{
Resumen
}

Una de las principales características del semiárido brasileño es la variabilidad climática y la escasez de agua. Vivir con la región semiárida, aún con todo su potencial naturalista y cultural, es un desafío, debido a los largos períodos de sequía y la limitación de los recursos hídricos. Este estudio tiene como objetivo adecuar el Bosque Modelo como metodología de solución de conflictos en acciones de manejo participativo en el territorio del Polo Borborema para fortalecer coaliciones y medidas estratégicas de convivencia con la región semiárida. Por tanto, se utilizó la metodología Bosque como modelo para la resolución de conflictos a través de un corte espacial o paisajístico de un escenario en desequilibrio ambiental y social. Así, los principales resultados obtenidos mediante la aplicación del modelo forestal fueron las diversas alternativas posibles para la resolución de conflictos a través de la movilización social con diferentes intereses y, en consecuencia, el fortalecimiento de las medidas encaminadas a mitigar los efectos del proceso de desertificación en una región con escasez. de los recursos naturales y está experimentando un proceso de extinción de su bioma, la Caatinga. Por tanto, se concluye que fue posible obtener una mejor visualización de la gestión participativa en el territorio de Borborema. Cabe señalar que los posibles conflictos que surgen de diferentes grupos también se discuten como medidas de la efectividad de la gestión participativa.

Palabras clave: Semiárido; Movilización social; Coaliciones.

\section{Introdução}

O semiárido brasileiro apresenta grandes variabilidade climática e desta forma, conta com a escassez de água em algumas áreas. Conviver com o semiárido mesmo com todo o seu potencial naturalístico e cultural é um desafio difícil e árduo devido os prolongados e severos períodos de estiagens e a limitação dos recursos hídricos acabam a limitar o desenvolvimento econômico (Peruzzo, 2015).

O Semiárido brasileiro é marcado pelo déficit hídrico com índice pluviométrico entre 200 e 800 mm ao ano, distribuído irregularmente no tempo e no espaço, além de apresentar uma taxa de evaporação (mais de 3 mil mm anuais) maior do que a de precipitação devido ao alto índice de insolação por um longo período (IBGE, 2010). Essa região possui algumas características físicas que não são favoráveis ao seu desenvolvimento socioeconômico da população, tal como o solo raso, rocha cristalina e déficit hídrico. No entanto, o bioma Caatinga ainda assim é muito resistente aos períodos prolongados de estiagem e consegue adaptar-se e desenvolver-se as condições naturais. Sendo assim, o longo período de estiagem não é o único e exclusivo motivos para o crescimento desenfreado do processo de desertificação no semiárido brasileiro (Sá et al., 2010).

Na Paraíba, dezesseis municípios estão inseridos na faixa de zoneamento do semiárido e encontram-se na região do 
Agreste e microrregiões do Brejo, Cariri e Curimataú as quais apresentam um baixo índice de acúmulo pluviométrico. Os sindicatos rurais desses municípios fazem parte de uma rede de articulação que se chama Pólo da Borborema (antigo Pólo Sindical da Borborema) um movimento sindical composto por 21 municípios e associações que debatem estratégias e políticas públicas de convivência com o semiárido. O Pólo da Borborema é o ator político e social frente ao cenário de fortalecimento na melhoria de vida das famílias agricultoras (Peruzzo, 2015).

A partir das rodas de diálogos com os agricultores, associações, ONG's e entidades governamentais são apresentados diferentes tipos de conflitos de interesses. Que em muitos casos, sem possível solução, ocasionado a vulnerabilidade e o enfraquecimento de algumas coalisões que encontram na gestão participativa uma possibilidade de apoio nos períodos de estiagem prolongada e de convivência com a seca. (Silva et al., 2016).

O Bosque Modelo é uma perspectiva metodológica utilizada para solucionar conflitos através de um recorte espacial ou paisagístico de um cenário em desequilíbrio ambiental e social. para tanto, a gestão participativa que é uma característica ascendente no território da Borborema é possível uma forma sistêmica para o combate à desertificação, fortalecimento da agricultura familiar, soberania, segurança alimentar e acesso as políticas de recursos hídricos (Santos \& Pereira, 2018).

Este trabalho justifica-se pela necessidade de metodologias que busquem alternativas possíveis para solução de conflitos através da mobilização social com diversos interesses e consequentemente o fortalecimento de medidas que visem atenuar os efeitos do processo de desertificação em uma região com escassez de recursos naturais e que vive um processo de extinção de seu bioma, a Caatinga.

O presente estudo visa adequar o Bosque Modelo como metodologia de solução de conflitos nas ações de gestão participativa no território do Pólo da Borborema para o fortalecimento das coalisões e medidas estratégicas de convivência para com o semiárido.

\section{Fundamentação teórica}

\subsection{O Semiárido Brasileiro e a Convivência com a Seca}

A intervenção do Estado Brasileiro na região Nordeste sempre foi marcada pela centralização e fragmentação de suas ações e políticas públicas, se concretizando pela criação de organismos de "luta" contra a seca (Passador et al., 2010). Ao longo da história de formação territorial do semiárido nordestino, foram criados diversos órgãos como a Inspetoria de Obras Contra a Seca (IOCS), a Inspetoria Federal de Obras Contra as Secas (IFOCS) e o Departamento Nacional de Obras Contra as Secas (DNOCS) (Araújo et al., 2015).

O Governo Federal vem apoiando esta nova forma de enfrentar o problema das secas, através de novos programas, tais como o Programa Um Milhão de Cisternas (P1MC) e o Programa Uma Terra Duas Águas (P1+2) (Araújo et al., 2015).

\subsection{Pólo da Borborema: Um dos Principais Atores na Gestão Participativa para o Desenvolvimento Rural}

O Polo da Borborema, corresponde a uma articulação de sindicatos rurais e organizações de apoio à agricultura familiar no território da Borborema, na Paraíba. Uma das características fundamentais desse território foi existência prévia de uma articulação regional em território, distinguida por forte presença da sociedade civil com grande capacidade de ação coletiva e de iniciativas locais (Diniz, 2007).

Formado por uma rede de 15 sindicatos de trabalhadoras e trabalhadores rurais (STRs), aproximadamente 150 associações comunitárias e uma organização regional de agricultores ecológicos, o Polo da Borborema vem apoiando redes locais de inovação agroecológica que articulam mais de 5 mil famílias agricultoras dos 15 municípios que conformam o Território da Borborema (Silveira et al., 2010).

O Território da Borborema teve sua homologação realizada em 29 de julho de 2003, após a constituição do Programa 
dos Territórios Rurais de Identidade da Secretaria de Desenvolvimento Territorial e Ministério do Desenvolvimento Agrário (SDT/MDA).

Primeiro, o território SDT inclui o município urbano de Campina Grande e, segundo seu espaço geográfico é contínuo, seguindo as normas estabelecidas pela SDT. Os municípios do território da SDT que não participam do Pólo Sindical são os municípios de atuação do Fórum dos Assentados da Reforma Agrária (Borborema, Serrania, Pilões, Areia), localizados ao leste do território do Pólo Sindical, assim como alguns municípios isolados, localizados no Sudeste (Serra Redonda), no Oeste (Puxinanã) e no Noroeste (Algodão de Jandaíra) (Piraux \& Bonnal, 2007).

Os municípios localizados na parte ocidental do "território" do Pólo Sindical não foram incorporados no território da SDT da Borborema, mas sim no território da SDT do Cariri (Piraux \& Bonnal, 2007).

Analisando a partir de seu lugar na história do território, pode-se dizer que o Pólo retoma e atualiza uma longa tradição de resistências sociais que aí se estruturaram como respostas ativas a conjunturas políticas e econômicas adversas à agricultura camponesa (Silveira et al., 2010).

\subsection{Autonomia Sustentável das Famílias Agricultoras e a Convivência com Seca}

De acordo com Silva, (2003) a perspectiva da convivência requer e implica um processo cultural, de educação, de uma nova aprendizagem sobre o meio ambiente, dos seus limites e potencialidades. Ou seja, a convivência envolve a percepção da complexidade e requer uma abordagem sistêmica do semiárido brasileiro possibilitando a compreensão das dimensões geofísica, social, econômica, política e cultural. No que se refere à necessidade e possibilidade de convivência com os ecossistemas frágeis, a construção da sustentabilidade depende de um processo participativo da população local no resgate e construção cultural de alternativas apropriadas aos ecossistemas (Silva, 2003). Neste sentido, é de fundamental importância o resgate dos conhecimentos das populações locais, somando-se aos demais saberes acumulados pela humanidade sobre as ocorrências da natureza (Silva, 2003; Silva, 2018).

A irregularidade dos índices pluviométricos, as altas taxas de evapotranspiração e os longos períodos de seca são fatores que tornam a água um fator limitante na região semiárida (Vidal \& Neto, 2014). Desta forma, torna-se viável para o combate dessa problemática o reúso da água residuária doméstica na agricultura como alternativa estratégica para a produção de alimentos visando o desenvolvimento da atividade econômica baseadas na agricultura principalmente de cunho familiar, utilizando o instrumento de reuso de água como proposta para solucionar ou anemizar da escassez hídrica nas regiões áridas e semiáridas do Brasil (Amaral et al., 2017).

Na maioria das residências do semiárido brasileiro, a reutilização da água para agricultura de cunho familiar, além de impulsionar a agricultura, torna possível também a prática de atividades mitigadores para uma posterior sustentabilidade hídrica (Tucci, 2017). A despejo proveniente do uso da água nas residências, inclusive a contribuição do vaso sanitário, portanto o reuso da água contribui para a sustentabilidade, mas por si só não garante que seja sustentável (Zerbinatti, 2011).

Estabelecimento de um aproveitamento racional e ecologicamente sustentável da natureza em benefício das populações locais, levando-as a incorporar a preocupação com a conservação da biodiversidade aos seus próprios interesses, como um componente da estratégia de desenvolvimento (Sachs, 2000).

São diversos os motivos que levam ao aumento do uso de águas residuais para a agricultura, impulsionado em parte pela crescente escassez e degradação de recursos hídricos na região semiárida brasileira, pelo aumento da população e pelas quais o mal uso desse recurso (Binati, 2017). Portanto, essa técnica de reutilização de águas já utilizadas no uso de águas domesticas, tem como principal proposito assegurar a sustentabilidade hídrica e ambiental para a população e ao meio ambiente (Silva \& Orsine, 2011). 


\section{Metodologia}

\subsection{Perspectiva metodológica do Modelo Bosque na convivência com o semiárido.}

Bosque Modelo é um processo de base social em que grupos que representam uma diversidade de atores trabalham conjuntamente visando o desenvolvimento sustentável de uma paisagem ou território onde a floresta desempenha um papel importante (Floresta, 2014). De acordo com o IMF de 2008 o conceito do Bosque Modelo resume-se aos aspectos de paisagens, parcerias, compromissos com a sustentabilidade, governanças, programa de atividades e compartilhamento de conhecimentos, capacitações e networking.

A aplicação Bosque Modelo para cada diferente cenário é um processo transparente, democrático e geralmente baseado em consenso, em que as regras para o processo de parceria são definidas pelos parceiros de rede nacional de nível superior. Através da troca eficaz de informações, experiências e ideias, o Bosque Modelo aprende a extrair forças e consegue adaptar-se em diferentes cenários de forma eficaz (Naysmith, 2003). Essa capacidade de adaptar-se em diferentes realidades dá-se ao modelo de Bosque Modelo a autonomia de gerir os recursos naturais de forma coerente, examinando as interações entre a atividade humana e o mundo natural, desenvolver capacidades locais para o manejo florestal sustentável (sfm), incorporar conhecimento tradicional e envolvimento aborígine no manejo florestal de manejo incorporar vários valores no SFM e, por sua vez, vincular o SFM à tecnologia, desenvolver amplas parcerias funcionais para o desenvolvimento sustentável, demonstrar manejo florestal sustentável em terras públicas e privadas, medir e avaliar o progresso em direção ao SFM e comunicar com o público em geral. (Naysmith, 2003).

Os autores (Angelstam, Elbakidze, Axelsson. et. out, 2019) propõem seis princípios a serem seguidos para aplicação do Bosque Modelo que são:

Princípio 1: Uma paisagem grande o suficiente para representar a diversidade de uma área usos e valores da floresta;

Princípio 2: Uma parceria inclusiva e dinâmica;

Princípio 3: Um compromisso com a sustentabilidade;

Princípio 4: Uma estrutura de governança representativa, participativo, transparente e responsável.

Princípio 5: Um programa de atividades que reflete as necessidades dos parceiros e Valores;

Princípio 6. Um compromisso com o compartilhamento de conhecimento, a capacitação e networking em vários níveis.

O Modelo Bosque já foi aplicado no Projeto Bosque Modelo Caçador Inventário Florestal Santa Catarina. Também aplicado no Projeto Arboretum na Bahia, nesse sentido aplicação no processo de soluções de conflitos nas ações de gestão participativa do território da Borborema-Paraíba pode ser relevante para a gestão dessa área e para a solução de conflito internos.

\subsection{Caracterização da área de estudo}

O Território da Borborema ocupa uma área de $3.233 \mathrm{~km}^{2}$ (23,1\% do Estado de...) sendo composto por 21 municípios, como Areia, Areial, Borborema, Campina Grande, Casserengue, Esperança, Lagoa Seca, Massaranduba, Matinhas, Montadas, Pilões, Puxinanã, Queimadas, Remígio, São Sebastião de Lagoa de Roça, Serra Redonda, Serraria e Solânea. Sendo assim, estes 18 municípios fazem parte do território da Borborema em questão (Figura 1).

Estes municípios estão distribuídos em cinco microrregiões geográficas definidas pelo IBGE: Brejo Paraibano, Campina Grande, Curimataú Ocidental, Curimataú Oriental e Esperança (CAI, 2011). 
Figura 1 - Mapa Político dos Municípios do Polo da Borborema no ano 2000.

\section{Mapa Político dos Municípios do Polo da Borborema}

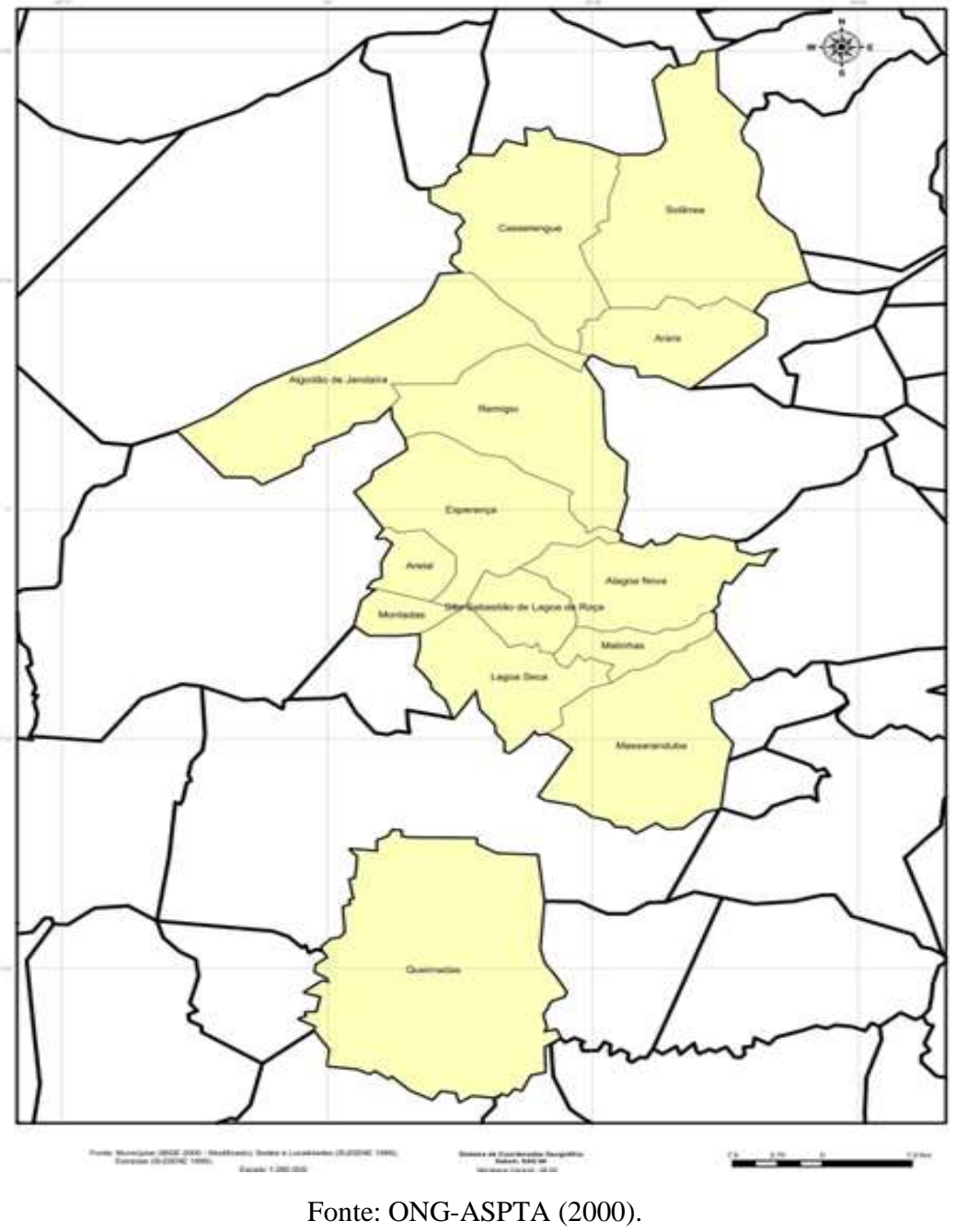

\subsection{Parcerias inclusivas e dinâmicas}

No que se refere ao território da Borborema os parceiros são: os sindicatos rurais dos 21 municípios que compreendem o território, Pólo da Borborema, ONG’S, Universidades, Órgãos governamentais (INSA, EMBRAPA, EMATER). Essas parcerias precisam ter objetivos em comum, exemplo, sustentabilidade, mesmo que apresente conflitos diversos. Além desses, outros grupos e organizações fazem parte do processo de solução de conflitos, priorizando a sustentabilidade.

Esses espaços representados na figura acima representam a cooperação de espaços governamentais e não governamentais, buscam a manutenção e o bem estar da natureza, preconizando o desenvolvimento sustentável das práticas econômicas realizadas no território da Borborema, como intuído de solucionar conflitos nas ações de gestão participativa. Alguns outros parceiros podem ser mesurados no modelo de bosque.

Projetos Parceiros executados pelo Modelo Bosque:

-O Rio do Peixe: educação ambiental, desenvolvimento de sistema de monitoramento hidrobiológico, avaliação da qualidade da água, capacitação de professores (Comitê da Bacia Hidrográfica do Rio do Peixe);

-SOS Nascentes: aumento do índice de saneamento no interior do município por meio da proteção de nascentes, tratamento de efluentes domésticos, educação ambiental (FUNDEMA);

-Cadastro Ambiental Rural (CAR): suporte técnico aos pequenos agricultores para inscrição no CAR (Polícia 
Ambiental);

-Pescar: resgaste e capacitação de adolescentes em situação de risco social em silvicultura, tecnologia da madeira, informática (Juliana Florestal e SENAI);

-Programa de Aquisição de Alimentos: produção de alimentos orgânicos por assentados da reforma agrária e comercialização (Cooperativa Mãos que Lutam de Caçador);

-Apicultura Sustentável: desenvolvimento e difusão de técnicas para aumento de produtividade e enquadramento nas normas certificadoras (Associação dos Apicultores de Caçador e região-ACAP.

\section{Resultados e Discussão}

\subsection{Compromisso com a sustentabilidade}

Nas rodas de diálogos entre os participantes os principais interesses sustentáveis defendidos são: práticas agroecológicas, soberania e segurança alimentar, fortalecimento da agricultura familiar, programa de cisternas, autonomia do pequeno agricultor (Costa, 2020). Todos precisam defender objetivos comuns, exemplo, sustentabilidade.

Embora as metas de sustentabilidade sejam um passo importante para a sustentabilidade dos recursos naturais, ainda há grandes dificuldades de pôr em pratica essa estratégia. As organizações públicas, privadas e não governamentais buscam juntas pelo compromisso ambiental (Vignati, 2020). Sendo assim, esses órgãos devem se concentrar no desenvolvimento de estratégias que visem motivar os demais setores econômicos a terem essa mesma preocupação e colocar em práticas medida mitigadoras para solucionar os problemas ambientais.

O compromisso de todos setores com a sustentabilidade ambiental tendem a contribuir para tal propósito porque os passageiros estão cada vez mais ambientalmente conscientes e educados e valorizam a sustentabilidade (Hülse; Ferrer; Demarchi, 2020).

\subsection{Uma estrutura de governança representativa, participativa, transparente e responsável.}

Através do Pólo da Borborema representado por sindicatos e associações é possível ver a implementação de políticas públicas que possibilitam a convivência com o semiárido. O propósito de esta associação é de criar um espaço de diálogo entre as partes interessadas para identificar problemas e coletar ideias para estabelecer uma estratégia de desenvolvimento para os setores que encontram-se com problemas a serem solucionados (Silveira \& Larvada, 2020). Desta forma, se faz necessário uma governança representativa com capacidade de propor medidas capazes de atenuar problemas de gestão no território da Borborema-Paraíba.

O processo participativo pode identificar governança problemas e critérios que precisavam ser avaliados e melhorados. Três interesses principais entre as partes interessadas ficaram claros: (1) para participar da tomada de decisões; (2) criar sinergias para o desenvolvimento sustentável de atividades econômicas e de desenvolvimento social; e (3) para colaborar na geração e troca de conhecimentos e experiências de governança complexa sistemas para melhorar a qualidade da governança (Ferreira, 2021). Sendo assim, identificou-se a necessidade de avaliar a qualidade da governança com o propósito de solucionar as problemáticas existentes nos indicadores sociais, econômicos e ambientais na região com um sistema participativo de abordagem colaborativa.

A partir de estudos, conhecimentos e algumas experiências trocadas por governante, agentes governamentais: Representantes eleitos e burocratas agindo como fiduciários, facilitadores e reguladores, que legislam e gerenciam o uso de recursos naturais com o intuito de participar de forma colaborativa na gestão e manutenção desses recursos, propiciando o desenvolvimento econômico e social com responsabilidades fiduciárias implícitas para sustentar e conservar os recursos naturais para benefício da sociedade sem causar danos preocupantes (Basto, 2020). 
Desta forma, governança representativa, participativa, transparente e responsável busca através de subconjuntos informados e interessados de propriedades públicos que através do interesse de melhor e desenvolver a região compartilham algumas responsabilidades científicas e sociais formais. Por sua vez, conduzindo pesquisas voltadas para o desenvolvimento da região, sendo relevantes para as políticas, comunicando a ciência aos tomadores de decisão e de gestão, e educando o público (Paro, 2017).

Vale salientar, que as organizações não governamentais (ONGs), são informados e interessados subconjunto de proprietários públicos que compartilham algumas responsabilidades científicas e sociais informais, financiando (e, às vezes, conduzindo) relevantes para a política pesquisa pesqueira, comunicando ciência para gestão e política tomadores de decisão e educar o público Comunidades de interesse: Subconjuntos de proprietários públicos com interesses comuns e poder de voto (Hülse; Ferrer; Demarchi, 2020). Neste estudo, os autores abrangeram partes interessadas envolvidas em diferentes estudos científicos relacionados com o bem governança e sustentabilidade na região de território da Borborema-Paraíba.

\subsection{Programa de atividades que reflete as necessidades dos parceiros e Valores.}

Dentre programas pode-se destacar com ênfase as tecnologias sociais hídricas TSH (Tecnologia Sociais Hídricas) vem desempenhando papel muito importante na redução das desigualdades sócias e principalmente regionais, pois, são capazes de dar autonomia ao meio rural, através da geração de renda e uso adequado dos recursos naturais no semiárido (Sousa et al., 2018).

O P1MC é um dos maiores programas de TSH construído no estado da Paraíba, assim como o P1+2, ele representou um salto sem precedentes na qualidade de vida das populações que habitam o semiárido Paraibano e consequentemente o brasileiro, onde possibilitou diversas famílias que não tinham acesso a água a finalmente ter acesso a esse bem natural que é para todos (Sobreira, 2018).

O principal foco deste programa é o acesso à água em qualidade, quando fala em quantidade, visando o consumo imediato nas residências familiares onde foi implantado, levando em consideração que essa pode ser a única forma de acesso a água possível para diversas famílias (Gomes; Heller, 2016). Por ser a tecnologia primária, este serviu de teste e validação para as tecnologias que vieram em sequência. Iniciado nos anos 2000, com incentivos da AECID - Agência Espanhola de Cooperação Internacional para o Desenvolvimento, junto a ASA. Este programa já construiu mais de 60.000 cisternas, em 184 municípios do estado (Figura 2), atendendo cerca de $80 \%$ de todos os municípios Paraibanos (Azevedo, 2017). 
Figura 2 - Cisterna usada na captação da água da chuva no município de Cubati-PB.

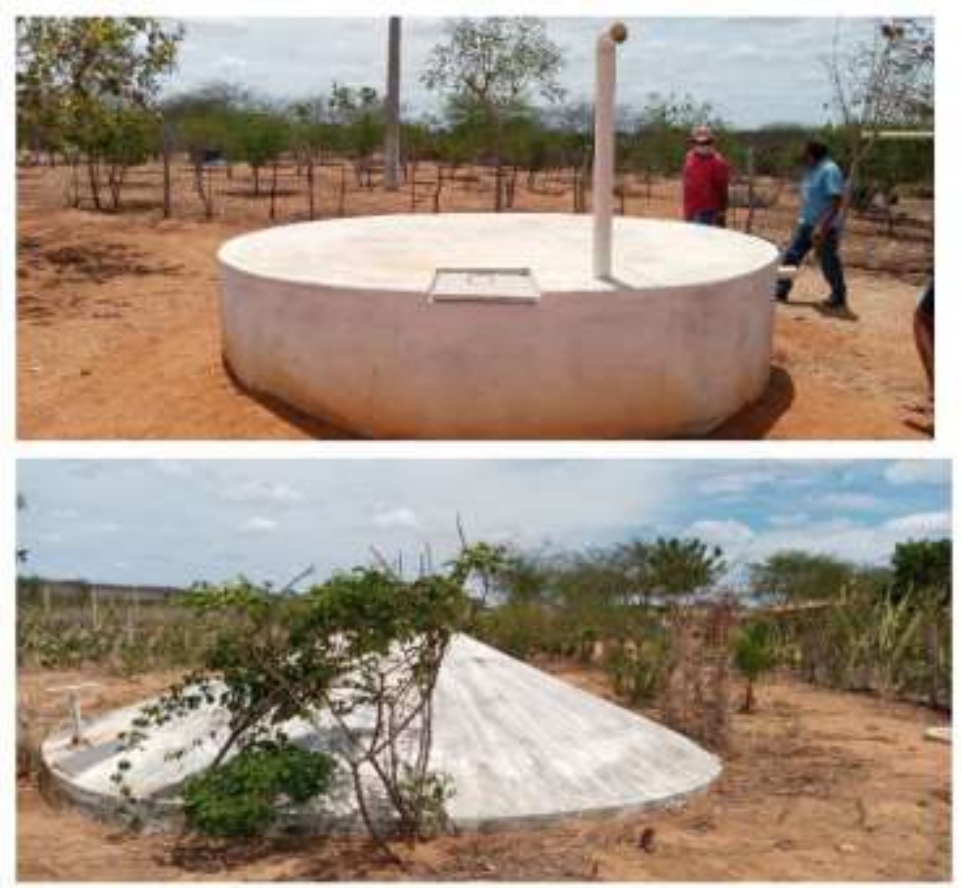

Fonte: Autores (2021).

A vantagem dessas tecnologias é que elas são muito baratas, chegam na ponta e permitem um aproveitamento minucioso da água e dos solos. Não havendo desperdício nem agressão ao meio ambiente. (Zanella, 2014). O programa tem como objetivo, garantir água de boa qualidade, gerando um uso racional desse recurso de tal modo que sua escassez relativa não continue a constituir impedimento ao desenvolvimento sustentável da região (Almeida \& Falção, 2020).

Outro ponto importante do programa é a capacitação da população, a qual passa a ter o domínio necessário da técnica para captação e armazenamento da água. Fazendo com que a qualidade da alimentação e da água melhora, com desdobramentos positivos na saúde e na renda (Zanella, 2014). Diante das tecnologias sociais hídricas destaca-se o P1+2 sendo atualmente reconhecida e discutida na academia, com um vasto acervo sobre suas formas de uso e aplicação, auxiliando na validação e incentivo a expansão da implantação, possibilitando a difusão da técnica como instrumento que auxilia famílias carentes da zona rural que por sua vez, não tem acesso água no período de seca (Ferreira, 2019).

O P1+2 inicialmente se resumia a construção de uma segunda tecnologia, que fosse agregada as famílias que inicialmente tiveram acesso ao P1MC. O principal foco desta tecnologia era a construção de um segundo reservatório, desta vez de $52 \mathrm{~m}^{3}$ e totalmente voltado para a água de produção, sendo esta para pequenas irrigações e dessedentação animal (Candiotto et al., 2016). Está tecnologia possibilitou um salto na qualidade de vida, e ao mesmo tempo acesso a meios de escoamento e comercialização da produção, via programas governamentais como o PAA - Programa de Aquisição de Alimentos e PNAEPrograma Nacional de Alimentação Escolar, conforme apresentado por Ferreira, (2019).

Essas Tecnologias Sociais tem o objetivo de realizar mudanças nas estruturas sociais das populações do semiárido, tendo como base um processo simples de captação e armazenamento de água da chuva, visando o abastecimento de residências no período de estiagem (Batista, 2010).

Nos assentamentos da Paraíba há famílias que, ao utilizarem essas tecnologias e práticas agrícolas, garantem sua alimentação e obtêm uma renda muito superior a qualquer programa assistencial do governo. Além do mais, se ganha em cidadania, já que as pessoas passam a viver do fruto do seu trabalho. Há ainda o fator ambiental: evitam-se as pragas e a salinização dos solos, que tornam inviável a atividade da irrigação em muitos projetos. (Montenegro, 2012). 


\section{Conclusão}

A partir dos princípios aplicados oriundos do Bosque Modelo foi possível obter uma melhor visualização da gestão participativa no território da Borborema. É preciso destacar que os possíveis conflitos que surgem dos diferentes grupos passam a ser discutidos também como medidas de eficácia da gestão participativa. Ora, se o fortalecimento dessa gestão se dá pela mobilização social é imprescindível a inserção da sociedade fazendo com que o processo seja dinâmico e criativo para pensar nas medidas adotadas de combate ao processo de desertificação e possibilidade de convivência. O recorte espacial do território possibilita uma análise mais aprofundada acerca do bioma Caatinga que nos dias atuais sofre com ameaças de extinção devido as estiagens prolongadas e ações antrópicas. Além disso, os diversos conflitos que acontecem no processo de participação social e governança dos recursos naturais precisam atender de forma comum os critérios de sustentabilidade e a solução ser compartilhada através de rodas de diálogos analisando a conjuntura e contexto atual.

\section{Referências}

Amaral Cunha, A. P. M., de Barros Brito, S. S., Rossato, L., dos Santos Alvalá, R. C., Cunningham, C., Zeri, M., \& Carvalho, M. A. (2017). Avaliação de indicador para o monitoramento dos impactos da seca em áreas de pastagens no semiárido do Brasil. Revista Brasileira de Cartografia, 69(1).

Almeida, C. L.; \& Falcão, J. S. (2020). Convivência com o semiárido a partir do uso de cisternas de placas no município de Frecheirinhas, estado do Ceará, Brasil. Agua y Territorio/Water and Landscape, (15), 89-100.

Araújo, F. V; Vianna, p. C; Souza, M. C. S; \& Ruffo, C. L. M. (2015). Célula de Acompanhamento e Informação o Território da Borborema-CAI. Relatório Analítico e Parcial. Revista Geosaberes, 6(2), $168-177$.

Azevêdo, Andrea Carla de. (2017). Autonomia X Dependência: políticas de água no Semiárido e desenvolvimento regional. Tese de Doutorado. 00500: Universidade de Coimbra.

Batista, J. B. V., Carlotto, M. S., Coutinho, A. S., \& Augusto, L. G. D. S. (2010). Prevalência da Síndrome de Burnout e fatores sociodemográficos e laborais em professores de escolas municipais da cidade de João Pessoa, PB. Revista brasileira de epidemiologia, 13, $502-512$.

Binati, C. R. A. (2017). Mudança climática e recursos hídricos: desafios e contribuições dos planos diretores dos municípios da bacia hidrográfica Billings na região metropolitana de São Paulo. 2017. 308 f. Dissertação (Arquitetura e Urbanismo) - Universidade Presbiteriana Mackenzie, São Paulo.

Burgos, A., \& Mertens, F. (2016). As redes de colaboração no turismo de base comunitária: implicações para a gestão participativa. Tourism \& Management Studies, 12(2), 18-27.

Candiotto, Luciano Zanetti Pessôa; Grisa, Felipe Fontoura; Schimitz, Luiz Antonio. (2016). Considerações sobre a experiência de construção de cisternas em unidades de produção e vida familiares (UPVFs) do Município De Francisco Beltrão-PR/Considerations about cisterns construction's experience in family life and production units (FLPU) of Fra. Revista Nera, (29), 174-193.

Costa Arsky, I. (2020). Os efeitos do Programa Cisternas no acesso à água no semiárido. Desenvolvimento e Meio Ambiente, 55.

Diniz, P. C. O. (2007). Da experimentação social ao “experimentalismo institucional”. Trajetórias de relações entre Estado e sociedade civil: experiências no Semiárido. Campina Grande (PB), Tese de Doutorado. CH-UFCG/UFPB-CCHLA/PPGS, setembro, 230 p.

EMBRAPA Florestas. (2014). Bosque Modelo Caçador: Oportunidade para gestão territorial participativa. Simpósio Nacional de Inventário Florestal.

Ferreira, Fernanda Barbosa. (2019). Desenvolvimento regional e políticas públicas de incentivo à inovação: os Núcleos de Inovação Tecnológica (NIT) nas universidades públicas da Paraíba. 2019. 95f. Dissertação (Programa de Pós-Graduação em Desenvolvimento Regional - PPGDR) - Universidade Estadual da Paraíba, Campina Grande - PB.

Ferreira, C. D. (2021). Participação de grupos de interesse na formulação de políticas públicas: alinhamento dos objetivos de desenvolvimento sustentável ambiental com o planejamento orçamentário do governo federal do Brasil.

Gomes, U. A. F., \& Heller, L. (2016). Acesso à água proporcionado pelo Programa de Formação e Mobilização Social para Convivência com o Semiárido: Um Milhão de Cisternas Rurais: combate à seca ou ruptura da vulnerabilidade? Engenharia Sanitária e Ambiental, 21, 623-633.

Hülse, L., Ferrer, G. R., Demarchi, C., \& dos Santos, A. M. (2020). Indissociabilidade entre sustentabilidade e Escolas Criativas e suas implicações para religar o ensino à complexidade da vida. Revista Polyphonía, 31(1), 67-84.

IBGE, Instituto Brasileiro de Geografia e Estatística. Censo Demográfico de 2010, 2010.

IMFN, 2008. Model Forest Development Guide. International Model Forest Network Secretariat, Ottawa. Canada, 34 p.

Montenegro, A. A. A; \& Montenegro, S. M. G. L. (2012). Olhares sobre as políticas públicas de recursos hídricos para o semiárido. IN: Recursos hídricos em regiões semiáridas / editores, Hans Raj Gheyi, Vital Pedro da Silva Paz, Salomão de Sousa Medeiros, Carlos de Oliveira Galvão - Campina Grande, PB: Instituto Nacional do Semiárido, Cruz das Almas, BA: Universidade Federal do Recôncavo da Bahia, 258P.

Naysmith, J. K. R. P. F. (2003). Canada’s Model Forest Program-Building on Success. 0399-C3. Québec City, Canada. 
P. Angelstam, et al. Forest Policy and Economics 101 (2019) 96-110

Paro, V. H. (2017). Gestão democrática da escola pública. Cortez Editora.

Passador, C. S; passador, 1. J.; Huayta, V. R. (2010). Políticas Contra la Sequía e la Técnica de Cisterna en Brasil. Revista Agroalimentaria (Caracas), 16, 101113.

Peruzzo, C. M. K. (2015). Comunicação popular e comunitária em práticas de desenvolvimento rural na região de Borborema (PB-Brasil). Comunicação \& Sociedade, 37(2), 183-208.

Piraux, M.; Bonnal, P. (2007). Dinâmicas Territoriais, Projetos Coletivos e Multifuncionalidade da Agricultura Familiar na Região do Entorno de Campina Grande (Paraíba). Pesquisa e ações de divulgação sobre o tema da multifuncionalidade da agricultura familiar e desenvolvimento territorial no Brasil. Relatório 3.4. $43 \mathrm{p}$.

Sachs, I. (2000). Ecodesenvolvimento: crescer sem destruir. Tradução de Eneida Araújo. Vértice.

Santos, A. J. G., \& Pereira, J. A. (2018). Planejamento e implementação de estratégias por meio da gestão participativa entre diretores e associados do clube AABB De Naviraí-MS. Encontro Internacional de Gestão, Desenvolvimento e Inovação (EIGEDIN), 2(1).

Sá, I. B., Cunha, T. J. F., Teixeira, A. D. C., Angelotti, F., \& Drumond, M. A. (2010). Processos de desertificação no Semiárido brasileiro. Embrapa SemiáridoCapítulo em livro científico (ALICE).

Silva, R.M.A. (2003). Entre dois paradigmas: Combate a seca e Convivência com o semiárido. Revista Soc. estado. 18(1-2).

Silva, L. C.; Orsine, Joice Vinhal Costa. (2011). Reutilização de água como ferramenta de sistemas de gestão ambiental, agroindustriais e domésticos. Enciclopédia Biosfera, Centro Científico Conhecer-Goiânia, 7(13), 1280-1293.

Silva, L. R. D. (2018). Não me chame de mulata: uma reflexão sobre a tradução em literatura afrodescendente no Brasil no par de línguas espanhol-português. Trabalhos em Linguística Aplicada, 57, 71-88.

Silva, S. D., de Medeiros, V. P., \& da Silva, A. B. (2016). Tecnologias sociais hídricas para convivência com o Semiárido: o caso de um assentamento rural do município de Cabaceiras-PB. Holos, 1, 295-309.

Silveira, 1. M.; freire, a. G.; diniz, p. C. (2010). O pólo da borborema: ator contemporâneo das lutas camponesas pelo território. Revista agriculturas. 7(1), 13$19 \mathrm{p}$.

Silveira, B. R., \& Lavarda, R. A. B. (2020). Relação entre startup de base tecnológica e stakeholders à luz da estratégia como prática e teoria da saliência| relationship between startup of technological basis and stakeholders in the light of the strategy as practice and theory of salience. Revista Brasileira de Gestão $e$ Inovação (Brazilian Journal of Management \& Innovation), 8(1), 1-24.

Sousa, A. B., da Costa, C. T. F., Firmino, P. R. A., \& de Souza Batista, V. (2018). Tecnologias sociais de convivência com o Semiárido na região do Cariri cearense. Cadernos de Ciência \& Tecnologia, 34(2), 197-220.

Sobreira, D. N. (2018). Das muitas margaridas: A construção de um feminismo rural no estado da Paraíba, Brasil. Mediapolis-Revista de Comunicação, Jornalismo e Espaço Público, (7), 47-60.

Tucci, C. E. M. (2017). Indicador de sustentabilidade hídrica urbana. Revista Gestão de Água da América Latina, 14, 1-12.

Vidal, T. F., \& Capelo Neto, J. (2014). Dinâmica de nitrogênio e fósforo em reservatório na região semiárida utilizando balanço de massa. Revista Brasileira de Engenharia Agrícola e Ambiental, 18, 402-407.

Vignati, F. (2020). Gestão de destinos turísticos: como atrair pessoas para pólos, cidades e países. Editora Senac Rio.

Zanella, M. E. (2014). Considerações sobre o clima e os recursos hídricos do semiárido nordestino. Caderno Prudentino de Geografia, 1(36), $126-142$.

Zerbinatti, O. E. et al. (2011). Qualidade da água proveniente da chuva coletada em diferentes tipos de telhados. Engenharia Ambiental: Pesquisa e Tecnologia, $8(3)$. 